\title{
DESVIOS ANGULARES EM POTROS DA RAÇA CRIOULA NA REGIÃO SUL DO RS DO PRIMEIRO AO OITAVO MÊS DE VIDA SOB MANEJO EXTENSIVO DE CRIAÇÃO
}

\author{
Julio César Paganela, ${ }^{1}$ Carlos Anselmo dos Santos, ${ }^{1}$ Pedro Kutscher Ripoll, ${ }^{1}$ \\ Cahue da Rosa Paz, ${ }^{1}$ Carlos Eduardo Wayne Nogueira ${ }^{2}$ e David Alejandro González ${ }^{3}$
}

1. Acadêmicos de Medicina Veterinária, Faculdade de Veterinária, Universidade Federal de Pelotas, RS, Brasil

2. Médico veterinário, professor doutor do Departamento de Clínicas Veterinárias, Faculdade de Veterinária, Universidade Federal de Pelotas, RS, Brasil

3. Doutor em Epidemiologia, Programa de Pós-Graduação em Epidemiologia, Universidade Federal de Pelotas, Brasil.

\section{RESUMO}

A conformação dos membros dos potros, futuros atletas, tornou-se o alvo principal da atenção de veterinários e criadores nos últimos vinte anos, em virtude da contribuição de uma conformação deficiente na maior incidência de lesões músculo-esqueléticas e da consequentemente maior valorização econômica de indivíduos com aprumos corretos. O objetivo deste estudo é identificar as principais alterações angulares de potros da raça Crioula do primeiro ao oitavo mês de vida, destacando as de maior ocorrência. Foram avaliados 77 potros da geração 2007 , escolhidos por um processo de amostragem aleatória simples de um total de 164 potros criados nas propriedades analisadas. $\mathrm{O}$ estudo foi realizado em duas etapas: a primeira avaliação foi feita com animais entre um e quatro meses de idade e a segunda com os mesmos potros com idade entre cinco e oito meses. Com base nas duas avaliações realizadas, o principal desvio angular observado foi o desvio medial de pinças (estevado), sendo registrado em 47 potros. A identificação de alterações de desvios angulares após o nascimento ou seu surgimento é de fundamental importância, pois permite ao médico veterinário imediata intervenção, através da realização de casqueamento e/ou alterações no manejo ou cirurgia para correção dos desvios angulares.

PALAVRAS-CHAVES: Casqueamento, desvios angulares, potros, valgus.

\section{ABSTRACT}

\section{ANGULAR LIMB DEFORMITIES IN CRIOULO FOAL IN SOUTHERN REGION OF RIO GRANDE DO SUL, BRAZIL, FROM THE FIRST TO THE EIGHTH MONTH OF LIFE REARED UNDER EXTENSIVE MANAGEMENT}

The conformation of the limbs of foals, future athletes, has become the focus of attention of physicians and breeders in the last 20 years due to the contribution of a poor conformation to a higher incidence of musculoskeletal disorders and consequently greater economic recovery of individuals with correct angulations. The aim of this study was to identify the main angulation disorders of Crioulo foals from the first to the eighth month of life, highlighting those that are the most frequent. A total of 77 foals of the 2007 generation were evaluated, they were chosen by a process of simple random sampling of a total of 164 foals raised on the properties analyzed. The study was conducted in two stages, the first evaluation was performed with animals within 1 and 4 months of age and the second with the same foals aged 5 and 8 months. Considering the two evaluations, the main angular defect observed was toed-in, which was observed in 47 foals. The identification of defects in angulations after birth or its appearance is extremely important to the veterinary, allowing immediate intervention by performing trimming and/or changes in management or surgery for correction of angular deviation.

KEYWORDS: Angular deformities, foal, trimming, valgus. 


\section{INTRODUÇÃO}

As alterações de conformação são referidas como possíveis etiologias das lesões que acometem os equinos durante suas vidas desportivas. Dessa forma, as alterações do sistema locomotor são altamente representativas com alterações dos demais sistemas. São importantes não apenas nos primeiros meses de vida, quando normalmente se identificam, mas também na fase adulta, quando suas consequências podem dar origem a enfermidades graves, comprometendo o desempenho do animal e desperdiçando anos de investimento e trabalho (FREY et al., 2005).

A conformação dos membros dos potros, futuros atletas, tornou-se o alvo principal da atenção de veterinários e criadores nos últimos vinte anos, pois a conformação deficiente acarreta o aumento da incidência de lesões músculo-esqueléticas. Por isso, há maior valorização econômica de indivíduos com aprumos corretos (SANTSCHI, 2003). A incidência das claudicações é altamente influenciada pela distribuição do peso natural e pela distribuição do peso de acordo com o trabalho que o animal realiza (GODOY \& TEIXEIRA NETO, 2007).

A conformação é definida como a forma ou o contorno de um animal. Essa definição pode ser ampliada para incluir a relação de forma e função, em que os desvios angulares (DA) são os desvios do eixo vertical dos membros no seu plano frontal, denominando-se pelas articulações envolvidas. O desvio lateral e distal ao ponto de origem da alteração é denominado valgus e o desvio medial varus (AUER, 1992).

Já a posição de pinças para dentro, estevado, é aquela em que as pinças apontam uma em direção à outra quando vistas de frente, sendo que o membro pode estar torto desde sua origem no peito ou apenas da articulação metacarpofalangana para baixo (STASHAK, 2006). Na avaliação frontal, quando a linha de falanges está voltada lateralmente, a alteração é denominada esquerda.

Uma vez que os cavalos da raça Crioula são cavalos de sela e devem suportar o peso de um cavaleiro e ao mesmo tempo executar movimentos requeridos, assim, necessita-se de animais que tenham vida útil longa e saudável. É evidente, portanto, a importância de bons aprumos para a realização das atividades desempenhadas por esses animais.
O objetivo deste estudo é identificar as principais alterações angulares de potros da raça Crioula do primeiro ao oitavo mês de vida, destacando as alterações de maior ocorrência.

\section{MATERIAL E MÉTODOS}

Desenvolveu-se um estudo longitudinal e prospectivo com 77 potros da geração 2007, escolhidos por um processo de amostragem aleatória simples de um total de 164 potros criados em sete criatórios da região sul do Rio Grande do Sul. O estudo foi realizado em duas etapas, com uma avaliação feita com os animais entre um e quatro meses de idade e outra com os mesmos potros com idade entre cinco e oito meses. Todos os potros eram mantidos no sistema de manejo de cada propriedade, criando-se os potros somente a pasto e sem nenhum manejo de casqueamento.

Avaliaram-se somente os potros sem alterações clínicas detectáveis. Os animais eram colocados em estação sob superfície plana, para verificação visual da vista cranial e após vista caudal.

Para a avaliação e quantificação dos desvios angulares, empregou-se o seguinte critério: nenhum; 1 desvio; 2 desvios - tanto para os membros torácicos quanto para os pélvicos de cada animal. A mudança na frequência em ambos os períodos foi comparada mediante o teste de Wilcoxon para dados pareados. Repetiram-se as análises, considerando-se as categorias de idade em ambas as visitas (1-2,9 meses; 3-4,9 meses; 5-6,9 meses e $\geq 7$ meses). Todos os resultados foram repetidos de forma separada para machos e fêmeas. Empregou-se nas análises o pacote estatístico Stata 9.0, com grau de significância de 5\%.

\section{RESULTADOS}

Dos 77 potros avaliados, a proporção de fêmeas foi maior que de machos $(58,5 \%$ e $41,5 \%$, respectivamente).

A Figura 1 mostra redução estatisticamente significativa no número de DA do tipo valgus entre ambos os períodos de avaliação, que foi mais evidente para o tarsus valgus (frequência de $31,2 \%$ vs. $7,1 \%$ ) do que para o carpus valgus (frequência de $63,6 \%$ vs. 47,1).

Já para os DA dos tipos carpus varus e estevado anterior e posterior, não houve diferença esta- 
tisticamente significativa entre ambos os períodos de avaliação, sendo que o desvio estevado anterior teve frequência relativamente alta (aproximadamente 50\%). Os DA dos tipos esquerdo anterior apresentaram um padrão contrário entre ambos os períodos, sendo que o primeiro apresentou uma redução significativa (frequência de $24,7 \%$ vs. $8,6 \%$ ) e o segundo um aumento entre a primeira e a segunda visita (frequência de $6,5 \%$ vs. $17,1 \%)$.

Esses resultados foram similares aos encontrados na análise das categorias de idade (Figura 2), mostrando uma tendência inversa com os DA dos tipos carpus valgus, tarsus valgus e esquerdo anterior, uma tendência direta com o DA esquerdo anterior e nenhuma relação com os desvios carpus varus e estevados anterior e posterior.

$\mathrm{Na}$ estratificação do número de DA conforme o sexo, os resultados foram diferentes apenas para os tipos carpus valgus e tarsus valgus (Figura 3). Embora ambos os sexos tenham apresentado uma redução na frequência desses desvios, estes foram mais evidentes e com uma diferença estatisticamente significativa apenas nas fêmeas, enquanto que para os machos essa redução ficou no limite da significância estatística.

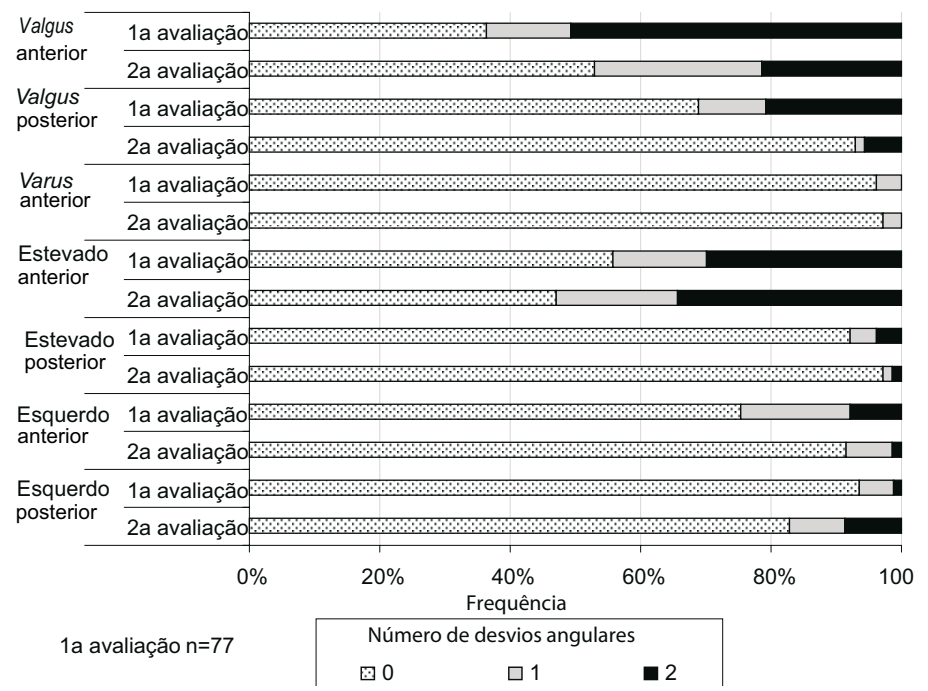

FIGURA 1. Frequência de desvios angulares em potros da raça Crioula em duas avaliações diferentes (1-4 meses e 5-8 meses). Rio Grande do Sul, 2008.

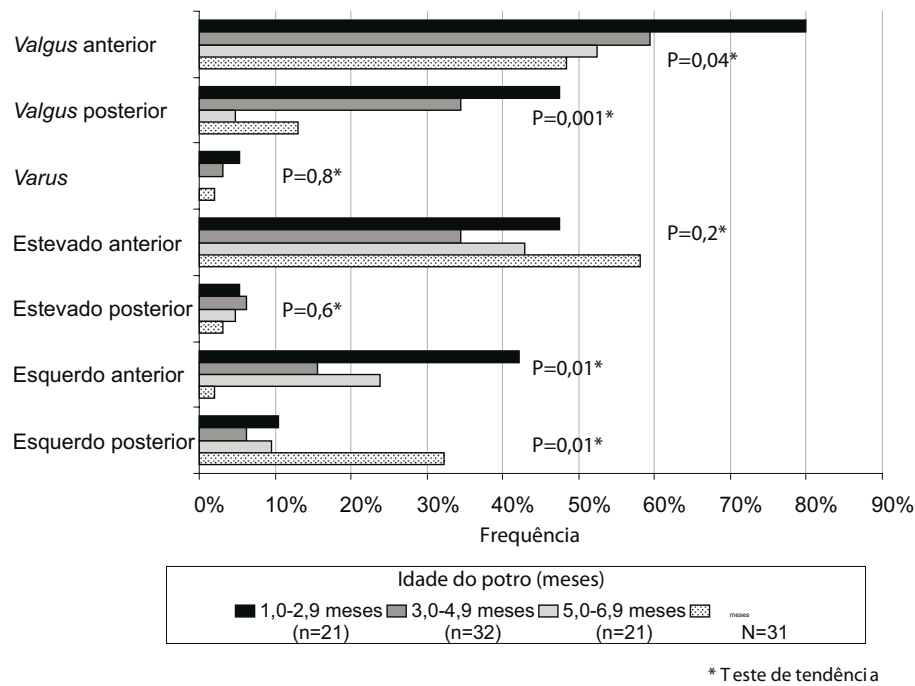

FIGURA 2. Presença de desvios angulares em potros da raça Crioula segundo grupos de idade. Rio Grande do Sul, 2008. 


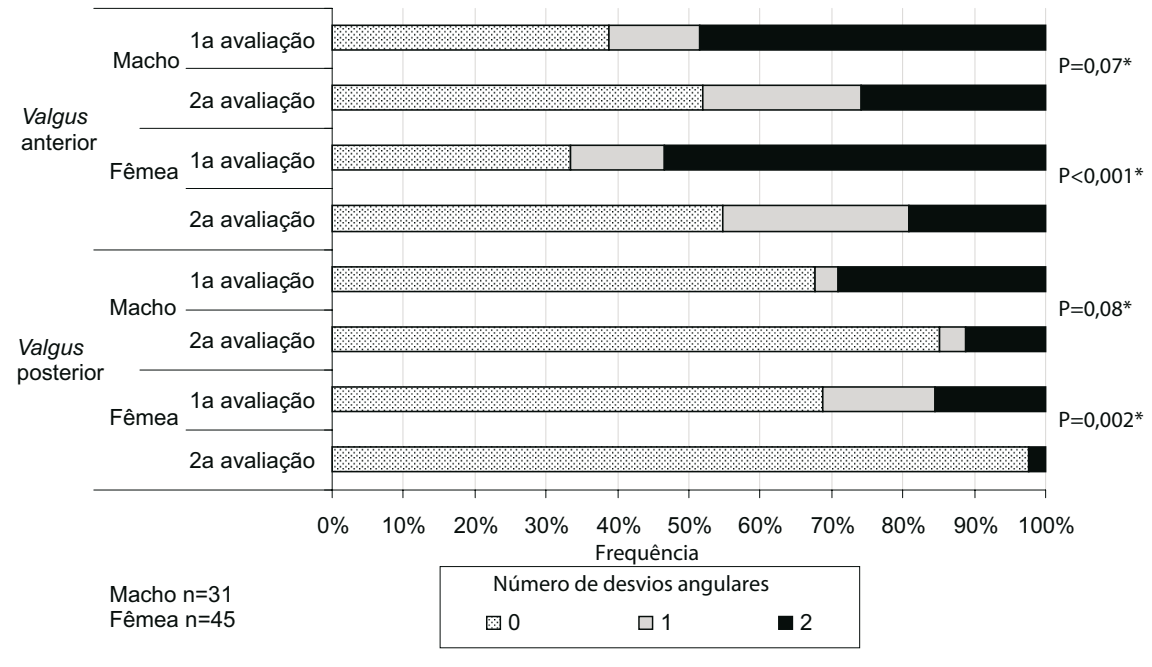

* Teste Wilcoxon para dados pareados

FIGURA 3. Frequência de desvios angulares do tipo Carpus Valgus em potros da raça Crioula segundo o sexo. Rio Grande do Sul, 2008 .

\section{DISCUSSÃO}

GREET et al. (2000) citam que potros neonatos de boa conformação devem apresentar um discreto desvio valgus do carpo de aproximadamente $2-5^{\circ}$, o qual se confirma com os resultados obtidos na segunda avaliação. Para BRAMLAGE \& EMBERTSON (1990), a correção natural progride até os quatro meses de vida. Uma leve correção natural ocorre entre os oito e dez meses, quando a placa de crescimento lateral apresenta crescimento maior, antes do seu fechamento, que ocorre ao redor de um ano de vida.

Em vista disso, a conformação comum para potros puro sangue de corrida desmamados é um leve desvio valgus, nas condições de carpo (RIECK, 2000). Porém alterações na conformação do carpo são importantes fatores predisponentes a fraturas dos ossos do carpo e injúrias nesta articulação (MUNDY, 1997).

Apesar de se ser baixa a frequência de alteração carpus varus entre os animais avaliados, ela se manteve na segunda avaliação, diferentemente da alteração carpus valgus, que diminuiu.

Neste trabalho, a presença de potros estevados entre a primeira e a segunda avaliação teve uma discreta redução e deve estar diretamente relacionada à conformação dos cavalos da raça Crioula. HOLMSTROM et al. (1990) encontraram uma frequência de 50\% de animais com conformação de pinças para dentro em grau leve nos cavalos de esporte de elite, indicando que mínimos desvios não impedem a integridade nem o desempenho.

Já o DA esquerdo teve aumento significativo da incidência entre a primeira e a segunda avaliação, podendo ser a questão genética e/ou não casqueamento dos animais o fator determinante.

Os DA de membros pélvicos, embora com pouca prevalência, devem ser levados em consideração, tendo em vista o tipo de exercício executado pelos animais da raça Crioula nas provas funcionais, em que os animais realizam paradas deslizantes e giros rápidos, com alterações abruptas de direção e esbarros longos. MUNDY et al. (1997) demonstram que os desvios angulares da articulação tibiotársica são citados como fatores predisponentes a alterações patológicas que levarão à queda de desempenho e a lesões. STASHAK \& HILL (2006) sugerem que as possíveis causas de claudicação serão artrite precoce de jarrete, síndrome do navicular e fratura de quartela.

A identificação de alterações de desvios angulares imediatamente após o nascimento ou seu surgimento é de fundamental importância, permitindo ao médico veterinário imediata intervenção. Outro fator determinante para correções dos desvios angulares é o casqueamento contínuo dos animais, que deve ser mantido como medida profilática contra futuros desvios.

Dessa forma, salienta-se, com o presente estudo, que as alterações do sistema locomotor do potro são determinantes para sua futura vida atlética e suas con- 
sequências podem dar origem a enfermidades graves, comprometendo, assim, o desempenho de toda uma criação.

\section{CONCLUSÃO}

Existem diversos fatores envolvidos para o desenvolvimento de deformidades angulares, sendo que os desvios podem regredir espontaneamente, como por exemplo a alteração carpus valgus. Entretanto, o desvio angular estevado não regrediu espontaneamente, sendo a principal alteração em potros da raça Crioula.

\section{REFERÊNCIAS}

AUER, J. A. Equine surgery. Filadélfia: Saunders, 1992.

BRAMLAGE, L. R.; EMBERTSON, R. M. Observations on the evalution and selection of foal limb deformities for surgical treatment. In: ANNU MEET OF AAEP, 37., 1990, Lexington, Kentucky. Proceedings... Lexington: American Association of Equine Practioners, 1990. p. 273-279. 454 p.

FREY, F. F.; NOGUEIRA, C. E. W.; RIPOLL, P. K.; SACCHET, R. S. Levantamento das alterações angulares e flexurais em potros na região de Bagé. In: CIC, 4., ENPOS, 7., 2005, Pelotas, RS. Anais... Pelostas, RS: Universidade Federal de Pelotas. 2005. Disponível em: <www.ufpel.edu.br/cic/2008/cd/pages/pdf/CA/CA_01945. pdf,>. Acesso em: ago. 2008.

GODOY, R. F., TEIXEIRA NETO, A. R. Claudicação em equinos. In: RIET-CORREA, F.; SCHILD, A. L.; LEMOS, R. A. A.;
BORGES, J. R. J. Doenças de ruminantes e eqüinos. 3. ed. Santa Maria, RS: Pallotti. 2007. p. 529-560.

HOLMSTROM, M.; MAGNUSUM L. E.; PHILIPSSEN, J. Variations in conformation of Swedish Warmblood horses and conformational characteristics of elite sport horses. Equine Veterinarian, v. 22, p. 186-193, 1990.

HUNT, R. J.; Management of angular limb deformities. In: ANUAL CONVENTION OF THE AMERICAN ASSOCIATION OF EQUINE PRACTITIONERS (AAEP), 46., 2000, Lexington. Procedeengs... Lexington, 2000. p. 128-129.

MARKS, D. Conformation and Soundness. In: ANUAL CONVENTION OF THE AMERICAN ASSOCIATION OF EQUINE PRACTITIONERS (AAEP), 46., 2000, Lexington. Proceedings... Lexington, 2000. p. 39-45.

MUNDY, G. D.; Review of risk factors associated with racing injuries. In: ANUAL CONVENTION OF THE AMERICAN ASSOCIATION OF EQUINE PRACTITIONERS (AAEP), 43., 1997, Lexington. Proceedengs... Lexington, 1997, p. 204-210.

RIECK, E. R.; DE LA CORTE, F. D.; SILVA, C. A. M.; BRASS, K. E. Desvios angulares em potros puro sangue de corrida do nascimento aos 30 dias de vida: origem e incidência. Ciência Rural, v. 30, n. 5 , p. $825-828,2000$.

SANTSCHI, E. M. Forelimb conformation in thoroughbred foals. In: ANNUAL AMERICAN COLLEGE OF VETERINARY SURGEONS SYMPOSIUM, 2003, Washington, MD. Proceeding... Bethesda: ACVS, 2003. p. 23-25.

STASHAK, T. S. Conformação e movimento: claudicação em equinos segundo Adams. 5. ed. São Paulo, SP: Roca, 2006. p. 55-59. 